\title{
Based on SARIMA-BP hybrid model and SSVM model of international crude oil price prediction research
}

Hua Luo ${ }^{1} \quad$ Xing $\mathrm{Liu}^{2} \quad$ Shuang Wang ${ }^{3}$

(Received 13 June 2016; revised 17 February 2017)

\begin{abstract}
We propose two hybrid prediction models for the international crude oil price: SARIMA-BP hybrid model; and SSVM model. The SARIMABP hybrid model combines seasonality analysis and autoregressive integrated moving average with back propagation neural network model. The SSVM model combines seasonality analysis with support vector machines. New York Mercantile Exchange (NYMEX) crude oil's monthly closing price, which ranges from January 2002 to April 2016, is selected as the experimental data sets. Experimental results are compared among the SARIMA-BP hybrid model, SSVM model and single SARIMA model. Empirical analysis shows that the SSVM model has highest
\end{abstract}

DOI:10.21914/anziamj.v58i0.10995, (c) Austral. Mathematical Soc. 2017. Published April 8, 2017, as part of the Proceedings of the 2016 Joint Conference of ANZIAM and Zhejiang Provincial Applied Mathematics Association. ISSN 1445-8810. (Print two pages per sheet of paper.) Copies of this article must not be made otherwise available on the internet; instead link directly to the DOI for this article. Record comments on this article via

http://journal. austms.org.au/ojs/index.php/ANZIAMJ/comment/add/10995/0 
prediction accuracy, and the single SARIMA model has lowest prediction accuracy. Thus, the SSVM model displays a better performance in oil price prediction. Further, the SSVM model predicts NYMEX crude oil's closing price will approach 50 dollars per barrel in May 2016.

Keywords: International crude oil; Price prediction; SARIMA-BP; SSVM; Prediction accuracy

\section{Contents}

1 Introduction

E144

2 Methodology

E147

2.1 Seasonality analysis . . . . . . . . . . . . . E147

2.2 ARIMA model . . . . . . . . . . . . . . . . . . . . . E E148

2.3 BP neural network . . . . . . . . . . . . . . . . . E149

2.4 SVM model. . . . . . . . . . . . . . . . . . E150

2.5 SARIMA-BP hybrid model and SSVM model . . . . . . . E151

3 Empirical analysis

E152

3.1 Experimental data and seasonality analysis . . . . . . E E152

3.2 Implementation of SARIMA-BP model . . . . . . . . . E154

3.3 Implementation of SSVM model . . . . . . . . . . . E156

3.4 Prediction results comparison . . . . . . . . . E156

4 Conclusions

E158

\section{Introduction}

Crude oil is a special kind of non-renewable and indispensable strategic resource to the survival and development of each country, known as the "blood 
of industry". Because of the important political influence of crude oil, it becomes a chase target of each country. Therefore, predicting the future crude oil price accurately not only provides powerful data to government, but also guarantees steady development of national economy and energy security. For crude oil, international general direction is divided into two research directions: quantitative and qualitative. Time series analysis is observing and studying time sequence, then finds its change law and trend, and predicts its future. Quantitative research in time series is very important. When we cannot find crucial factors that leads to data changes from so many factors, time series analysis method is very effective. Qualitative research mainly refers to the structure model: although it can explain the phenomenon of changes better in market supply and demand, its prediction is not very good.

Zhou et al. [18] examined the potential seasonality of China's crude oil import based on X-12 ARIMA, and the result showed seasonality existed. Pai et al. [11] proposed a hybrid methodology that exploited the unique strength of the ARIMA model and the support vector machine (SVM) model in stock price forecasting. Kriechbaumer et al. [7] considered the performance of ARIMA models in forecasting metal prices that was demonstrated to increase substantially through a wavelet-based multi-resolution analysis, which was prior to the ARIMA model. Koutroumanidis et al. [6] pointed out that ARIMA and artificial neural networks (ANN) hybrid model was used to predict the future selling prices of the wood that was produced by Greek state forest farms and the ARIMA-ANN hybrid model performed well. Mohammadi et al. [10] examined the usefulness of several ARIMA-GARCH model for modelling and forecasting the conditional mean and volatility of weekly crude oil spot prices in eleven international markets. These conclusions illustrate that ARIMA model can be used in time series prediction.

Neural network model can also be used in time series prediction. Chiroma et al. [3] proposed an alternative approach based on a genetic algorithm (GA) and neural network for the prediction of the West Texas Intermediate crude oil price. Laboissiere et al. [8] aimed at proposing a methodology that forecasted the maximum and minimum day stock prices of three Brazilian power distribu- 
tion companies by using ANNs method. Haya et al. [1] presented a novel type of recurrent neural network, and the regularized dynamic self-organized neural network inspired by the immune algorithm. Sampathkumar et al. [12] focused on the modelling and forecasting of land price in Chennai Metropolitan Area in the state of Tamilnadu, India used multiple regression and neural network techniques. Maran et al. [9] thought that modelling of ultrasound-assisted intensification of biodiesel production from neem (Azadirachta indica) oil was investigated using four factors and three level central composite rotatable design of response surface methodology. Singhal et al. [13] considered electricity price forecasting in deregulated open power markets using neural networks. Zeng et al. [16] found the prediction of Consumer Price Indexes and the power load of one district of China illustrated that prediction accuracy of $\operatorname{NNTFGM}(1,1)$ was higher than $\operatorname{TFGM}(1,1)$ for the fluctuating TF series.

A svM model can be used in time series prediction. Liwei et al. [4] employed independent component analysis to analyze crude oil price which was decomposed into several independent components corresponding to different types of influential factors affecting oil price. Wen et al. [14] used the singular spectrum analysis to decompose the stock price into terms of the trend, the market fluctuation, and the noise with different economic features over different time horizons, and then introduced these features into the SVM to make price predictions. Guo et al. [5] used GA to optimize the parameter selection methods of SVM in accordance with training data, and improved SVM forecasting precision. Zhang et al. [17] proposed a novel hybrid method to forecast crude oil price and found that the newly proposed hybrid method had a strong forecasting capability for crude oil price, due to its excellent performance in adaptation to the random sample selection, data frequency and structural breaks in samples.

Single models always perform poorly. This paper researches international crude oil price by using SARIMA-BP hybrid model and SSVM model. Two models are better than a single model. The SARIMA-BP model includes seasonality analysis, ARIMA, and BP neural network, while the SSVM model includes seasonality analysis and the SVM method. Section 2 discusses some 
theoretical aspects of the methodologies and useful modelling steps are discussed. Section 3 shows modelling process and prediction results of crude oil price through those models. Finally, Section 4 presents conclusions and some insights.

\section{Methodology}

Seasonality analysis, ARIMA, BP neural network and SVM models are summarized in this section as foundation to describe the SARIMA-BP hybrid model and SSVM model.

\subsection{Seasonality analysis}

In general, monthly data has seasonality. In order to facilitate subsequent modelling, seasonality of experiment data sets can be eliminated by using the seasonal index. Assume an observation sequence is $\left\{X_{t} \mid t=1,2, \ldots\right\}$. Now we define the seasonal index. Suppose an observation sequence of time series with seasonal effect is $\left\{X_{i j} \mid \mathfrak{i}=1,2, \ldots, L, j=1,2, \ldots, s\right\}$, here, $X_{i j}$ expresses the ith year (period) and $j$ th month (period) data. Then, the average of months (period) data is

$$
\bar{\chi}_{k}=\frac{1}{L} \sum_{i=1}^{L} X_{i j}, \quad j=1,2, \ldots, s,
$$

and the average for the whole period is

$$
\bar{\chi}=\frac{1}{L s} \sum_{i=1}^{L} \sum_{j=1}^{s} x_{i j} .
$$

Thus, the seasonal index is

$$
S_{k}=\frac{\bar{x}_{k}}{\bar{x}}, \quad k=1,2, \ldots, s .
$$


Let

$$
Y_{i j}=X_{i j} / S_{j}, \quad i=1,2, \ldots L, \quad j=1,2, \ldots s .
$$

An new time series $\left\{Y_{t} \mid t=1,2, \ldots\right\}$ is obtained after eliminating the seasonality.

\subsection{ARIMA model}

Time series modelling method is primarily based on historical data. For non-stationary time series, ARIMA model is mainly applied. $\operatorname{ARIMA}(p, d, q)$ is called autoregressive integrated moving average model, and the model is

$$
\left\{\begin{array}{l}
\Phi(B) \nabla^{d} y_{t}=\Theta(B) \varepsilon_{t} \\
E\left(\varepsilon_{t}\right)=0, \quad \operatorname{Var}\left(\varepsilon_{t}\right)=\sigma_{\varepsilon}^{2}, \quad E\left(\varepsilon_{t} \varepsilon_{s}\right)=0, \quad s \neq t \\
E\left(y_{s} \varepsilon_{t}\right)=0, \quad \text { for all } s \leqslant t,
\end{array}\right.
$$

where $\nabla^{\mathrm{d}}=(1-\mathrm{B})^{\mathrm{d}}, \Phi(\mathrm{B})=1-\phi_{1} \mathrm{~B}-\cdots-\phi_{\mathrm{p}} \mathrm{B}^{\mathrm{p}}$ and $\Theta(\mathrm{B})=1-$ $\theta_{1} B-\cdots-\theta_{q} B^{q}$ are smoothly reversible coefficient polynomials about the $\operatorname{ARMA}(p, q)$ model where $p$ is the autoregressive order, $q$ is the moving average order, $\mathrm{d}$ is the differential order, $\phi_{1}, \phi_{2}, \ldots, \phi_{\mathrm{p}}$ are the regression coefficients, $\theta_{1}, \theta_{2}, \ldots, \theta_{\mathrm{q}}$ are the moving average coefficients, $\mathrm{B}$ is difference operator, and $\varepsilon_{\mathrm{t}}$ is a white noise sequence.

The modelling process of ARIMA model is divided into three parts. The first part is the original data preprocessing. The actual transaction data generally are non-stationary time series. Thus we need to make the sequence smooth in order to change the non-stationary time series into stationary time series. ARIMA model with d-order difference sequence can be made into stationary time series. The second part is the model recognition and model parameters estimation. In the process of defining order, there are mainly three kinds of methods which use autocorrelation figure, partial autocorrelation figure for a preliminary judgement of the order of the sequence and the SBC minimum 
order information or AIC criterion to determine final order. The AIC criterion and the SBC minimum order model expression is

$$
\begin{aligned}
& \mathrm{AIC}=n \log \left(\hat{\sigma}_{\varepsilon}^{2}\right)+2(p+q+1), \\
& \mathrm{SBC}=n \log \left(\hat{\sigma}_{\varepsilon}^{2}\right)+\log (n)(p+q+1),
\end{aligned}
$$

where $\mathrm{n}$ is the number of samples. The third part is an adaptive test of the model. After the model identification and parameters estimation, the model is established correctly if the model error passes the test of white noise. When the test is not passed, the model needs to be reset and we determine the order and parameter estimation again.

\subsection{BP neural network}

A neural network is a kind of 'intelligent' information processing method. In numerous neural network structures, based on error back propagation neural network (BP), the neural network is a function of nonlinear differential weights training multi-layer network. The main idea of BP neural network is to use the least square method on the basis of negative gradient descent learning algorithm to modify weights and thresholds.

BP neural network mainly consists of input layer, hidden layer, and output layer. The learning process has two parts: forward part and backward part. Forward propagation refers to the information from input layer through hidden layer and transmitted to output layer. Back propagation refers to an iteration to modify the weights of the neural connections until the error between the output and the desired output values reach the allowable range.

For a basic BP neural network, assuming that the input signal is $x_{i}(i=$ $1,2, \ldots, m)$, the hidden layer output signal is $y_{j}(j=1,2, \ldots, n)$, and the output signal is $z_{k}(k=1,2, \ldots, l)$. The weight of $x_{i}$ and $y_{j}$ is set to $\omega_{i j}$, and the connection threshold value is $\theta_{i}$. The weight of $y_{j}$ and $z_{k}$ is set to $\omega_{j k}$, and connection threshold value to $\theta_{j}$. The desired output of the output 
node is $\hat{z}_{k}$. The output neuron model is

$$
y_{j}=f\left(\sum_{i=1}^{m} \omega_{i j} x_{i}-\theta_{i}\right), \quad z_{k}=f\left(\sum_{j=1}^{n} \omega_{j k} y_{j}-\theta_{j}\right) .
$$

Where $f(\cdot)$ refers to the activation sigmoid function

$$
f(x)=\frac{2}{1+e^{-2 x}}-1 .
$$

Here, the model's error between the output value and the expected value is

$$
\Delta=\frac{1}{2} \sum_{k=1}^{l}\left(z_{k}-\hat{z}_{k}\right)^{2} .
$$

If $\Delta$ is not within specification error, then modify the weights and the thresholds to change the error term until the model's error $\Delta$ meets the specification.

\section{$2.4 \quad$ SVM model}

A support vector machine (SVM) is based on linear classification. But you can imagine that not all data could be linear, such as two categories of point in the two-dimensional space may need a curve to differentiate their border. The principle of SVM is that points in low dimensional space mapped to a high-dimensional space may make them become linear separably. Using the principle of linear classification to determine classification boundary in high dimensional space, it is a kind of linear classification. In the original data space it is a kind of nonlinear classification. However, when scholars discuss the SVM algorithm, it is not discussed how to define the low dimension to the high dimensional space mapping algorithm (implied the algorithm) in its "nuclear function", apart from the optimisation problem ( finding the optimal solution of a target). 
The SVM classification plane is $\left(w^{\top} \cdot x\right)+b=0$, where $x$ is a multidimensional vector, $w^{\top}$ is a weight vector, and $b$ is the offset. The reciprocal of teh classification interval is $\frac{1}{2}\|w\|^{2}=\frac{1}{2}\left(w^{\top} \cdot w\right)$. So the optimisation problem [2] is

$$
\begin{aligned}
& \min _{\alpha} \frac{1}{2} \sum_{i=1}^{l} \sum_{j=1}^{l} y_{i} y_{j} \alpha_{i} \alpha_{j}\left(x_{i} \cdot x_{j}\right)-\sum_{j=1}^{l} \alpha_{j}, \\
& \text { such that } \quad \sum_{i=1}^{l} y_{i} \alpha_{i}=0, \quad \alpha_{i} \geqslant 0 .
\end{aligned}
$$

Calculate $\alpha^{*}$ (the optimal solution), then calculate the optimal solution

$$
w^{*}=\sum_{i=1}^{l} y_{i} a_{i}{ }^{*} x_{i} b^{*}=y_{j}-\sum_{i=1}^{l} y_{i} \alpha_{i}\left(x_{i} \cdot x_{j}\right) .
$$

This problem is a quadratic programming problem, which includes Lagrange function, duality theory and constraints.

\subsection{SARIMA-BP hybrid model and SSVM model}

Here, we propose the SARIMA-BP hybrid model and thessvm model. The SARIMA model combines seasonality analysis, ARIMA model with a BP neural network. Usually, an observation sequence $\left\{X_{t} \mid t=1,2, \ldots\right\}$ is divided into two parts which are linear autocorrelation structure $\left\{\mathrm{L}_{\mathrm{t}} \mid \mathrm{t}=1,2, \ldots\right\}$ and nonlinear structure $\left\{\mathrm{N}_{\mathrm{t}} \mid \mathrm{t}=1,2, \ldots\right\}$ :

$$
X_{t}=L_{t}+N_{t} .
$$

The part $\mathrm{L}_{\mathrm{t}}$ is modelled with ARIMA, and the other part $\mathrm{N}_{\mathrm{t}}$ modelled with a BP neural network. The modelling steps of SARIMA-BP hybrid model are the following. 
1. Get an new time series $\left\{Y_{t} \mid t=1,2, \ldots\right\}$ after eliminating the seasonal of $\left\{X_{t} \mid t=1,2, \ldots\right\}$ using the seasonal index.

2. Use ARIMA to model $\left\{Y_{t} \mid t=1,2, \ldots\right\}$ and its forecasting. Let the predicted result be $\hat{\mathrm{L}}_{t}$. Then we get the residual series $\left\{\boldsymbol{e}_{t}\right\}$ about $\left\{Y_{t} \mid t=1,2, \ldots\right\}$ and $\hat{L}_{t}$, that is $e_{t}=Y_{t}-\hat{L}_{t}$.

3. The series $\left\{e_{t}\right\}$ contains the nonlinear structure in the original sequence. We pose $e_{t}=f\left(e_{t-1}, e_{t-2}, \ldots, e_{t-n}\right)+\varepsilon$, where here $\varepsilon$ is a random error term. We construct the BP neural network of the series $\left\{e_{t}\right\}$, and predict the sequence by a built neural network. The predicted result is $\hat{e}_{t}$.

4. Then combine the predicted result of ARIMA with the predicted result of the BP neural network. Let $\hat{Y}_{t}=\hat{L}_{t}+\hat{e}_{t}$. Then we get the final predicted result $\hat{X}_{t}$ by multiplying the seasonal index.

The SSVM model combines seasonality analysis with SVM. The SVM method is based on statistical learning theory, vC dimension theory, and structure risk minimum principle. According to the complexity of model and learning ability, we try to seek a best kernel function on limited samples, then get the best generalisation ability.

\section{Empirical analysis}

\subsection{Experimental data and seasonality analysis}

We research NYMEX crude oil, and the crude oil points to the United States which is the New York Mercantile Exchange crude oil spot contracts. We take the monthly closing price of NYMEX crude oil as experimental data from January 2002 to April 2016, which is collected in China Eastern Airlines Futures trading software. Figure 1 plots the original closing price of crude oil. The figure shows the trend and seasonality. It is not smooth. What is 
Figure 1: International crude oil's monthly closing price sequence diagram.

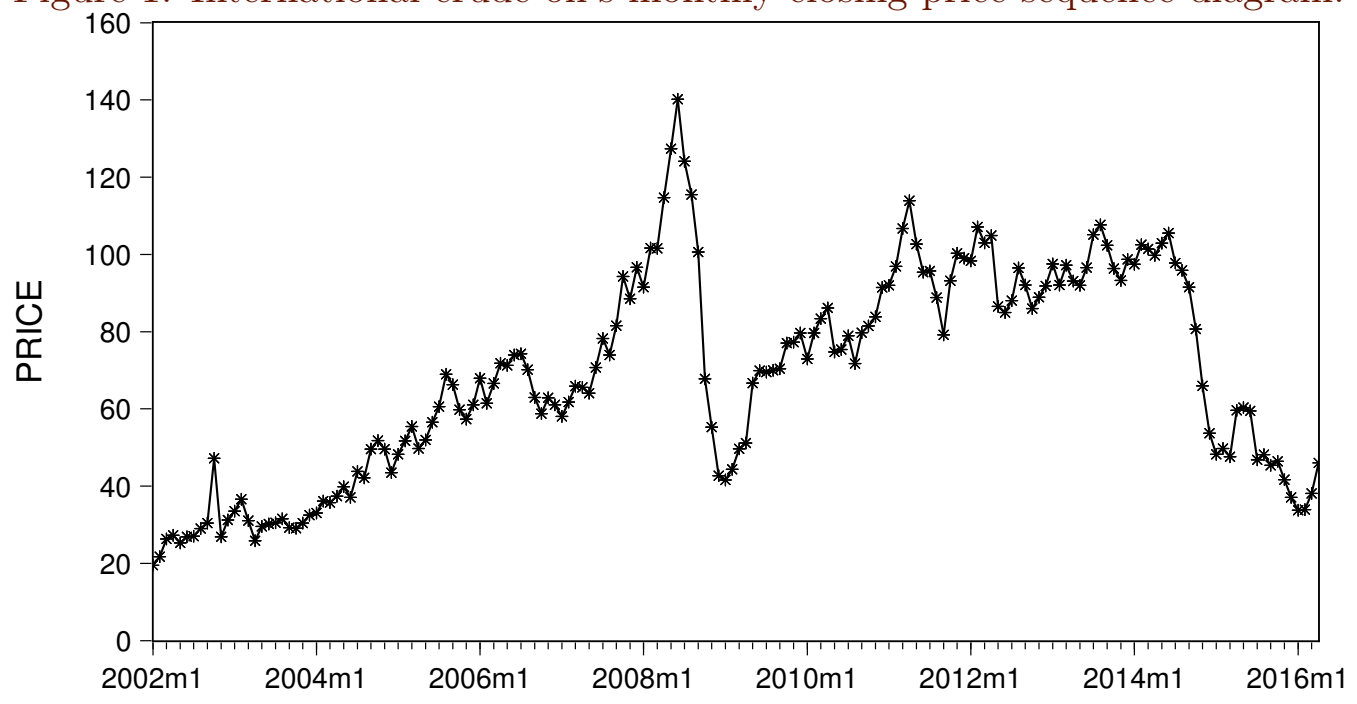

Table 1: Seasonal index.

\begin{tabular}{ccccccc}
\hline $\mathrm{k}$ (month) & 1 & 2 & 3 & 4 & 5 & 6 \\
$\mathrm{~S}_{\mathrm{k}}$ & 0.9296 & 0.9711 & 1.0002 & 1.0304 & 1.0245 & 1.0528 \\
\hline $\mathrm{k}($ month $)$ & 7 & 8 & 9 & 10 & 11 & 12 \\
$\mathrm{~S}_{\mathrm{k}}$ & 1.0505 & 1.0396 & 1.0101 & 0.9983 & 0.9493 & 0.9471 \\
\hline
\end{tabular}

more, according to the ADF test (unit root test) results, it accepts the null hypothesis. Thus, the original time series has an unit root.

Here, we take seasonality analysis for oil closing price and eliminate the seasonality of the original sequence by using seasonal index. Table 1 lists the derived seasonal index. 
Figure 2: First-order difference sequence diagram of NYMEX crude oil's monthly closing price.

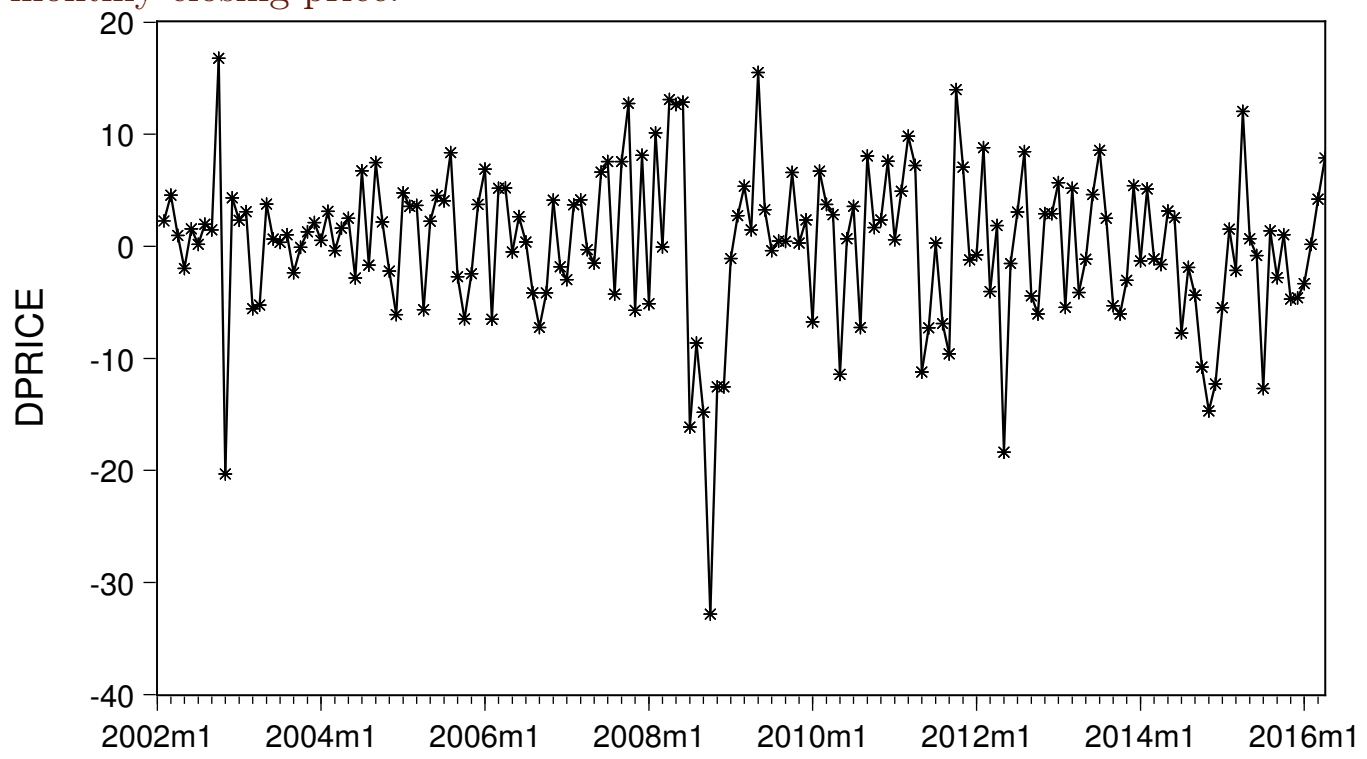

\subsection{Implementation of SARIMA-BP model}

The time series is non-stationary, but the difference operation may convert it to stationary. So next we consider the first order difference time series of original crude oil: shown in Figure 2. From the figure, the price of crude oil differences fluctuates at near zero and have a certain stability. Under a significance level of 0.05 , the Chi-squared test value of the delayed six order sequence is 0.5534 , which is significantly greater than 0.05 , so the sequence passes through a white noise test. Thus the price differences of crude oil is smooth.

According to the autocorrelation function and partial autocorrelation function diagram, the first order difference sequence is a regression model. The concrete model of order number requires an information criterion to determine the minimum. According to the corresponding model of the minimum information 
criterion, when $p=1$ and $q=0$ AIC and BIC are both minimum. Therefore, we determine NYMEX crude oil price time series in line with an ARIMA $(1,1,0)$ model.

Using a $\operatorname{ARIMA}(1,1,0)$ model to estimate the parameters of this model, the final model expression is

$$
\nabla Y_{\mathrm{t}}-0.1479=0.1755\left(\nabla \mathrm{Y}_{\mathrm{t}-1}-0.1479\right)+\varepsilon_{\mathrm{t}} .
$$

Here, $Y_{t}$ is closing price of crude oil at $t$ moment, $\nabla Y_{t}$ is first-order backward difference closing price of crude oil at $t$ moment, and $\left\{\varepsilon_{t}\right\}$ is a white noise sequence. Since $\nabla Y_{t}=Y_{t}-Y_{t-1}$, therefore

$$
Y_{t}=0.1219+1.1755 Y_{t-1}-0.1755 Y_{t-2}+\varepsilon_{t},
$$

After examination, the model passes the adaptability test, so this ARIMA $(1,1,0)$ model is adapted.

We apply $\operatorname{ARIMA}(1,1,0)$ model to forecast. Let the predicted result be $\hat{\mathrm{L}}_{\mathrm{t}}$. Then we get the residual series $e_{t}=Y_{t}-\hat{L}_{t}$. Next we construct a BP neural network for $\left\{\boldsymbol{e}_{\mathrm{t}}\right\}$.

The BP neural network input variables are $e_{t-3}, e_{t-2}$, and $e_{t-1}$, whereas the output variable is $e_{\mathrm{t}}$. Network training parameter settings [15] are as follows: the transfer function of input layer to hidden layer is tansig; the transfer function of hidden layer to output layer is purelin; the momentum coefficient is 0.2 ; the maximum number of learning for 5000 ; the learning rate is 0.1 ; the network error between the output and the actual value is made of the mean square error. The initial weights and thresholds between $(1,1)$ obey that of a uniform distribution random number. We eventually determine the number of hidden layer neurons, after many experiments, to be nine. Therefore, we build the BP neural network structure to be $3-9-1$, and we predict $e_{t}$ through well built BP neural network. 
Figure 3: Original data and predicted data of NYMEX crude oil per month closing price.

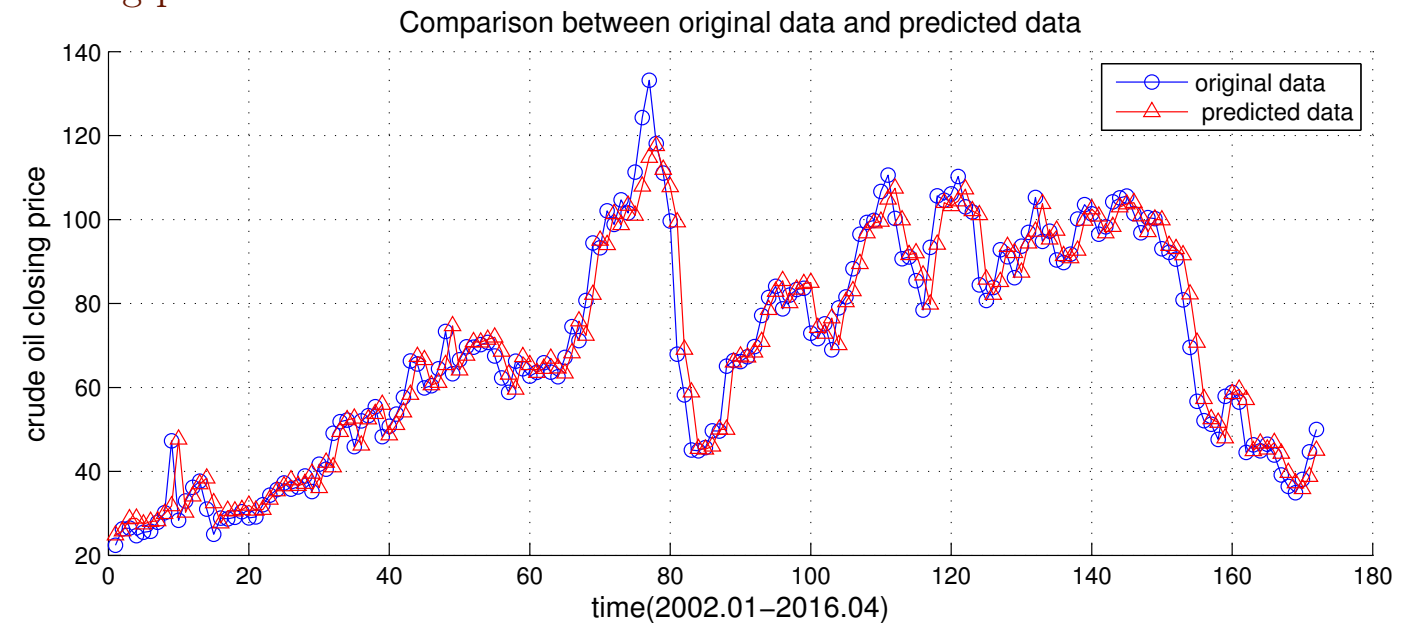

\subsection{Implementation of SSVM model}

Here we consider the series $\left\{Y_{t}\right\}$, which eliminate the seasonality of the original oil closing price series $\left\{X_{t}\right\}$. We use the SVM method and compute the results using MATLAB software. The results are shown in Figure 3.

\subsection{Prediction results comparison}

We list the prediction results of those models in Table 2. We use two kinds of error, one of error is MSD the mean square error, the other is MAD the average absolute error:

$$
\operatorname{MSD}=\frac{1}{n} \sum_{i=1}^{n}\left(a_{i}-\overline{a_{i}}\right)^{2}, \quad M A D=\frac{1}{n} \sum_{i=1}^{n}\left|a_{i}-\overline{a_{i}}\right| .
$$

Here, $\overline{a_{i}}=\frac{1}{n} \sum_{i=1}^{n} a_{i}$. The model error of the results are shown in Table 3. 
Table 2: Prediction results of those models among single SARIMA model, SARIMA-BP model and SSVM model.

\begin{tabular}{ccccc}
\hline Month & $\begin{array}{l}\text { Original } \\
\text { closing price } \\
\text { of crude oil }\end{array}$ & $\begin{array}{l}\text { Result of } \\
\text { single } \\
\text { SARIMA }\end{array}$ & $\begin{array}{l}\text { Result of } \\
\text { SARIMA-BP } \\
\text { hybrid model }\end{array}$ & $\begin{array}{l}\text { Result of } \\
\text { SSVM model }\end{array}$ \\
\hline 2015.07 & 46.77 & 44.69 & 46.92 & 46.33 \\
2015.08 & 48.16 & 48.61 & 48.28 & 48.60 \\
2015.09 & 45.36 & 45.23 & 45.49 & 45.84 \\
2015.10 & 46.39 & 46.78 & 46.51 & 46.79 \\
2015.11 & 41.68 & 41.36 & 41.83 & 41.96 \\
2015.12 & 37.07 & 36.39 & 37.42 & 36.79 \\
2016.01 & 33.74 & 33.41 & 34.30 & 33.90 \\
2016.02 & 33.90 & 33.75 & 34.64 & 33.60 \\
2016.03 & 38.11 & 38.79 & 38.56 & 38.33 \\
2016.04 & 46.00 & 47.30 & 46.38 & 46.49 \\
\hline
\end{tabular}

Table 3: Errors of those models among single SARIMA model, SARIMA-BP model and SSVM model.

\begin{tabular}{cccc}
\hline $\begin{array}{l}\text { Types of } \\
\text { error }\end{array}$ & $\begin{array}{l}\text { Result of } \\
\text { single } \\
\text { SARIMA }\end{array}$ & $\begin{array}{l}\text { Result of } \\
\text { SARIMA-BP } \\
\text { hybrid model }\end{array}$ & $\begin{array}{l}\text { Result of } \\
\text { SSVM model }\end{array}$ \\
\hline MSD & 0.7487 & 0.1126 & 0.0431 \\
MAD & 0.6340 & 0.2902 & 0.1815 \\
\hline
\end{tabular}


Table 3 shows that the MSD and MAD error of SSVM model is lowest in three models, and the error of single SARIMA model is highest. To a certain extent, it shows that the prediction accuracy of the SSVM model is highest and this model is more suitable than other models in international crude oil research. Comparing the single SARIMA with the SARIMA-BP hybrid model, we find that the hybrid model improves prediction accuracy effectively. That is to say, the SARIMA-BP hybrid model is better than the single SARIMA model. We also find the error of the SARIMA-BP hybrid model and the SSVM model is almost equal. In general, these two models are superior. At last, we use SSVM model to predict the closing price of NYMEX oil in the next few months, namely 50 dollars per barrel in May 2016.

\section{Conclusions}

We discussed SARIMA-BP hybrid model and SSVM model in research on the international crude oil price. We chose NYMEX crude oil closing price as the experimental data from January 2002 to April 2016. The empirical analysis shows that SARIMA-BP hybrid model and SSVM model both are better than single SARIMA model and have high prediction accuracy. But among SARIMABP hybrid, SSVM and single SARIMA those three models, the SSVM model is the best model because its prediction error is the lowest.

In addition, we used the SSVM model to predict the closing price of NYMEX oil in the next few months. The prediction is that the closing price of NYMEX oil will approach 50 dollars per barrel in May 2016. Such predictions could guide countries and investors. 


\section{References}

[1] Haya Al-Askar et al. "Regularized Dynamic Self Organized Neural Network Inspired by the Immune Algorithm for Financial Time Series Prediction". In: Lecture Notes in Computer Science 8590 (2014), pp. 56-62. DOI: 10.1007/978-3-319-09330-7_8 (cit. on p. E146).

[2] Chih Chung Chang and Chih Jen Lin. "LIBSVM: A library for support vector machines". In: Acm Transactions on Intelligent Systems \& Technology 2.3, article 27 (2007), pp. 389-396. DOI: 10.1145/1961189.1961199 (cit. on p. E151).

[3] Haruna Chiroma, Sameem Abdulkareem, and Tutut Herawan. "Evolutionary Neural Network model for West Texas Intermediate crude oil price prediction". In: Applied Energy 142 (2015), pp. 266-273. DOI: $10.1016 / \mathrm{j}$. apenergy.2014.12.045 (cit. on p. E145).

[4] Liwei Fan et al. "An ICA-based support vector regression scheme for forecasting crude oil prices". In: Technological Forecasting and Social Change 112 (2016), pp. 245-253. DOI: 10.1016/j.techfore.2016.04.027 (cit. on p. E146).

[5] Xiaopeng Guo, Da Cheng Li, and Anhui Zhang. "Improved Support Vector Machine Oil Price Forecast Model Based on Genetic Algorithm Optimization Parameters". In: Aasri Procedia 1.4 (2012), pp. 525-530. DOI: 10.1016/j.aasri.2012.06.082 (cit. on p. E146).

[6] Theodoros Koutroumanidis, Konstantinos Ioannou, and Garyfallos Arabatzis. "Predicting fuelwood prices in Greece with the use of ARIMA models, artificial neural networks and a hybrid ARIMAÍCANN model". In: Energy Policy 37.9 (2009), pp. 3627-3634. DOI: $10.1016 / \mathrm{j}$.enpol.2009.04.024 (cit. on p. E145).

[7] Thomas Kriechbaumer et al. "An improved waveletíCARIMA approach for forecasting metal prices". In: Resources Policy 39.1 (2014), pp. 32-41. DOI: $10.1016 / j$.resourpol.2013.10.005 (cit. on p. E145). 
[8] Leonel A. Laboissiere, Ricardo A. S. Fernandes, and

Guilherme G. Lage. "Maximum and minimum stock price forecasting of Brazilian power distribution companies based on artificial neural networks". In: Applied Soft Computing 35 (2015), pp. 66-74. DOI: 10.1016/j.asoc.2015.06.005 (cit. on p. E145).

[9] J. Prakash Maran and B. Priya. "Modeling of ultrasound assisted intensification of biodiesel production from neem ( Azadirachta indica) oil using response surface methodology and artificial neural network". In: Fuel 143 (2015), pp. 262-267. DOI: 10.1016/j.fuel.2014.11.058 (cit. on p. E146).

[10] Hassan Mohammadi and Lixian Su. "International evidence on crude oil price dynamics: Applications of ARIMA-GARCH models". In: Energy Economics 32.5 (2010), pp. 1001-1008. DOI: 10.1016/j . eneco.2010.04.009 (cit. on p. E145).

[11] Ping Feng Pai and Chih Sheng Lin. "A hybrid ARIMA and support vector machines model in stock price forecasting". In: Omega 33.6 (2005), pp. 497-505. DOI: 10.1016/j .omega.2004.07.024 (cit. on p. E145).

[12] V. Sampathkumar, M. Helen Santhi, and J. Vanjinathan. "Forecasting the Land Price Using Statistical and Neural Network Software". In: Procedia Computer Science 57 (2015), pp. 112-121. DOI: 10.1016/j.procs. 2015.07.377 (cit. on p. E146).

[13] Deepak Singhal and K. S. Swarup. "Electricity price forecasting using artificial neural networks". In: International Journal of Electrical Power \& Energy Systems 33.44 (2008), pp. 111-118. DOI: 10.1016/j.ijepes.2010.12.009 (cit. on p. E146).

[14] Fenghua Wen et al. "Stock Price Prediction based on SSA and SVM ą̂̀". In: Procedia Computer Science 31 (2014), pp. 625-631. DOI: 10.1016/j.procs. 2014.05.309 (cit. on p. E146). 
[15] S. E. Yang and L. Huang. "Financial crisis warning model based on BP neural network". In: Systems Engineering-theory \& Practice 25.1 (2005), pp. 12-19 (cit. on p. E155).

[16] Xiang Yan Zeng et al. "Triangular fuzzy series forecasting based on grey model and neural network". In: Applied Mathematical Modelling 40.3 (2015), pp. 1717-1727. DOI: 10.1016/j.apm.2015.08.009 (cit. on p. E146).

[17] Jin Liang Zhang, Yue Jun Zhang, and Lu Zhang. "A novel hybrid method for crude oil price forecasting". In: Energy Economics 49 (2015), pp. 649-659. DOI: 10.1016/j .eneco.2015.02.018 (cit. on p. E146).

[18] Zhong Bing Zhou and Xiu Cheng Dong. "Analysis about the seasonality of China's crude oil import based on X-12-ARIMA". In: Energy 42.42 (2012), pp. 281-288. DOI: 10.1016/j. energy. 2012.03.058 (cit. on p. E145).

\section{Author addresses}

1. Hua Luo, Department of Mathematics, College of Sciences, Zhejiang Sci-Tech University, Hangzhou 310018, China

mailto:luohuahill@163.com orcid:0000-0002-3272-3831

2. Xing Liu, Department of Mathematics, College of Sciences, Zhejiang Sci-Tech University, Hangzhou 310018, China

mailto:852410987@qq. com orcid:0000-0001-8065-4220

3. Shuang Wang, Department of Mathematics, College of Sciences, Zhejiang Sci-Tech University, Hangzhou 310018, China mailto:619458360@qq. com orcid:0000-0003-2343-5819 